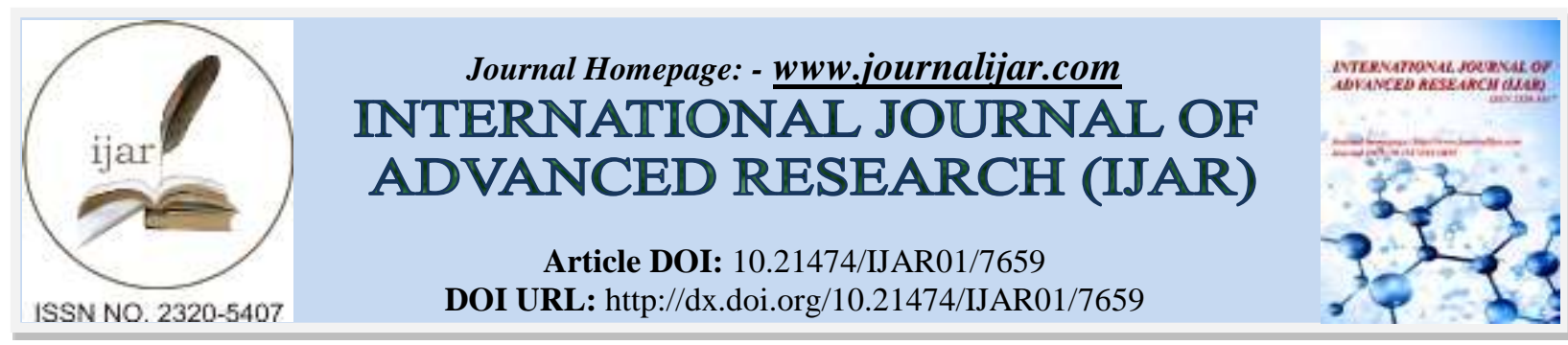

RESEARCH ARTICLE

\title{
BENIGN METASTASIZING LEIOMYOMA IN AN ELDERLY FEMALE, A CASE REPORT, AND REVIEW OF THE LITERATURE.
}

\author{
Hanan Alrammah ${ }^{1}$, Sara AlHajiri ${ }^{2}$, Abdelhaleem Bella ${ }^{3}$ and Hind Alsaif ${ }^{4}$. \\ 1. Medical Intern, King Fahad Hospital of the University, Imam Abdulrahman Bin Faisal University (IAU). \\ 2. Internal Medicine Resident, King Fahad Hospital of the University, IAU \\ 3. Department of Medicine, RCSI-Bahrain. ${ }^{4}$ Department of Radiology, college of medicine, Imam Abdulrahman \\ Bin Faisal University, Dammam, Saudi Arabia.
}

\section{Manuscript Info}

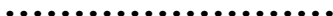 \\ Manuscript History}

Received: 03 July 2018

Final Accepted: 05 August 2018

Published: September 2018

Keywords:-

Benign Metastasizing Leiomyoma, lung nodules, uterine leiomyoma.

\section{Abstract}

Benign Metastasizing Leiomyoma (BML) is a rare disease mostly seen among women in the fertile period with a previous history or coincidental finding of uterine leiomyoma. We report a case of pulmonary BML from the uterus in a 73-year-old woman who underwent myomectomy at the age of 55 years. Two years later she had multiple nodules identified in the lung fields by computed chest tomography. Pathological examination of lung biopsy confirmed the diagnosis of BML with a uterine origin, and she was treated with Tamoxifen. She represented with 2 months history of dyspnea, cough and chest pain. It is the fourth reported case in the Middle East, we discuss the course of the patient and discuss the clinical presentation and management of BML.

Copy Right, IJAR, 2018,. All rights reserved.

\section{Introduction:-}

Benign Metastasizing Leiomyoma (BML) is a rare disease mostly encountered in females of reproductive age with a previous history or coincidental finding of uterine leiomyoma. This disease was first described by Steiner in 1939 , he reported a case of a patient who died due to metastasis of BML. Although benign, this disease may metastasize to various organs including the lungs, thereby causing fatal complications. The authors report a case of pulmonary BML from the uterus in a 73-year-old Saudi female. It is the fourth reported case in the Middle East, the authors discuss the course of the patient and discuss the clinical presentation and management of BML.

\section{Case presentation:-}

In 2015 a 73-Year-old nulliparous female was referred to King Fahd Hospital of the University (KFHU) for evaluation multiple pulmonary nodules seen on CT-Chest. She presented to a peripheral hospital with recurrent symptoms of dyspnoea, right-sided chest pain, and cough and was treated for presumed recurrent lower respiratory tract infections. Since 2013 she had been treated as bronchial asthma.

On review of her medical history it was found that she was seen in 1997 at age of 57 when presented with dyspnoea, right-sided chest pain and cough along with fever, chills, sweating, decrease in appetite, weight loss, and urinary urgency. The patient had a history of myomectomy for uterine leiomyoma in 1995 at age of 55 years. The physical examination then was unremarkable. Chest CT showed multiple well-defined opacities of varying sizes in the lungs. Bronchoscopy with bronchial lavage and biopsy were negative for malignant cells. Thoracotomy, with biopsy, was 
done and the results were consistent with Benign Metastasizing leiomyoma with uterine origin. The patient was discharged home with Tamoxifen the duration of which is not clear. Her recent evaluation showed decreased air entry on the right lower zone.

Her recent investigations showed multiple nodules in the lung of variable sizes with one cavitating (Figure-1) with newer and enlarging nodules compared with old images, the largest seen in the right hilar region, and small mediastinal lymph nodes. The pelvic ultrasound showed multiple uterine lesions, the largest one measuring $22 \times 16$ $\mathrm{cm}$ (Figure.2).

The blood chemistry and tumor markers including carcinoembryonic antigen (CEA), human chorionic gonadotropin (HCG), C125 and alpha-fetoprotein (AFP) were within normal range. Erythrocyte sedimentation rate(ESR) was mildly elevated $(37 \mathrm{~mm} / \mathrm{hr})$. The patient was referred to the obstetrics and gynecology department for follow up of the new uterine lesions and given an appointment for follow up on the pulmonary clinic. The patient refused the gynecological assessment and defaulted on the follow-up. The presumptive diagnosis is a recurrence of her uterine leiomyomata and with new pulmonary lesions due to the BML.

\section{Discussion:-}

In 1939, Steiner described the first case of BML, as a tumor composed of benign-appearing, well-differentiated smooth muscle cells and dense connective tissue ${ }^{1}$ Benign metastasizing leiomyoma (BML) is a rare medical condition which affects females in their reproductive age, with a mean age of presentation of 48 years ${ }^{2}$. Usually, these patients present with uterine Leiomyoma (UL) treated with hysterectomy or myomectomy. Uterine leiomyoma is the commonest gynecological neoplasm in women of child-bearing age with a prevalence rate of about $50 \%$. The metastasis may occur within a period ranging from 3 months to 26 years ${ }^{2}$

Being a benign tumor, the incidence of metastasis is extremely rare, however, it may occur. The lungs are the most affected extra uterine organ ${ }^{1}$. BML has been also described in lymph nodes, deep soft tissues, omentum and mesentery, bone, spine, skull base, and heart.

Both BML and UL share most importantly estrogen and progesterone receptors, the same immunohistochemical profile (p53-negative, BCL-2-positive) and a low proliferation index ${ }^{1}$, identical patterns of androgen receptor allelic inactivation $^{3}$, and their immunoreactivity for the HMB-45 antibody. Video-assisted thoracoscopic (VAT) wedge resection is the best method to harvest tissues for diagnosis ${ }^{4}$. As for the treatment of BML, There is no agreed consensus. In the reported cases, most premenopausal women underwent total hysterectomy \pm Bilateral SalpingoOophorectomy for the primary tumor, and to surgical resection \pm hormonal therapy in the form tamoxifen, progesterone, luteinizing-hormone-releasing hormone analog, and estrogen receptor modulators for the metastatic lesion, while observation was preferred in postmenopausal patients. In 2016, the first case of PBML in Saudi Arabia was reported by Alselwi WM et al. The patient's lesions were unrespectable, thus she was treated with hormonal therapy only and achieved a good clinical response ${ }^{5}$. Similarly, in our case, the patient was treated with Tamoxifen $(10 \mathrm{mg})$ twice daily for estrogen blockade. One study suggested that GnRH agonist could be used to decrease the disease burden in those who are not fit for surgical resection ${ }^{6}$, while another one proposed to consider Aromatase inhibitor and SERM as another option as a hormonal therapy for BML, especially for postmenopausal women ${ }^{7}$. Since the use of anti-estrogenic agents is associated with a decrease in bone marrow density, it is crucial to add bisphosphonates, calcium, and vitamin D and to measure bone density frequently. Left untreated, Ponea AM reported that complications such as the formation of cystic structures, destruction of lung parenchyma, and hemothorax which may lead to respiratory failure can occur in Pulmonary $\mathrm{BML}^{8}$.

\section{Conclusion:-}

Albeit very rare cause of multiple pulmonary nodules, BML should be kept in mind as one of the differential diagnosis in a female patient with a previous history of UL, and thorough medical history is important for that to avoid misdiagnosis and overtreatment. When diagnosed, BML should be treated, to avoid its fatal complications. Despite there is no standard management algorithm for the treatment of BML, its positivity for estrogen and progesterone receptors makes anti-hormonal therapy a valid option. 


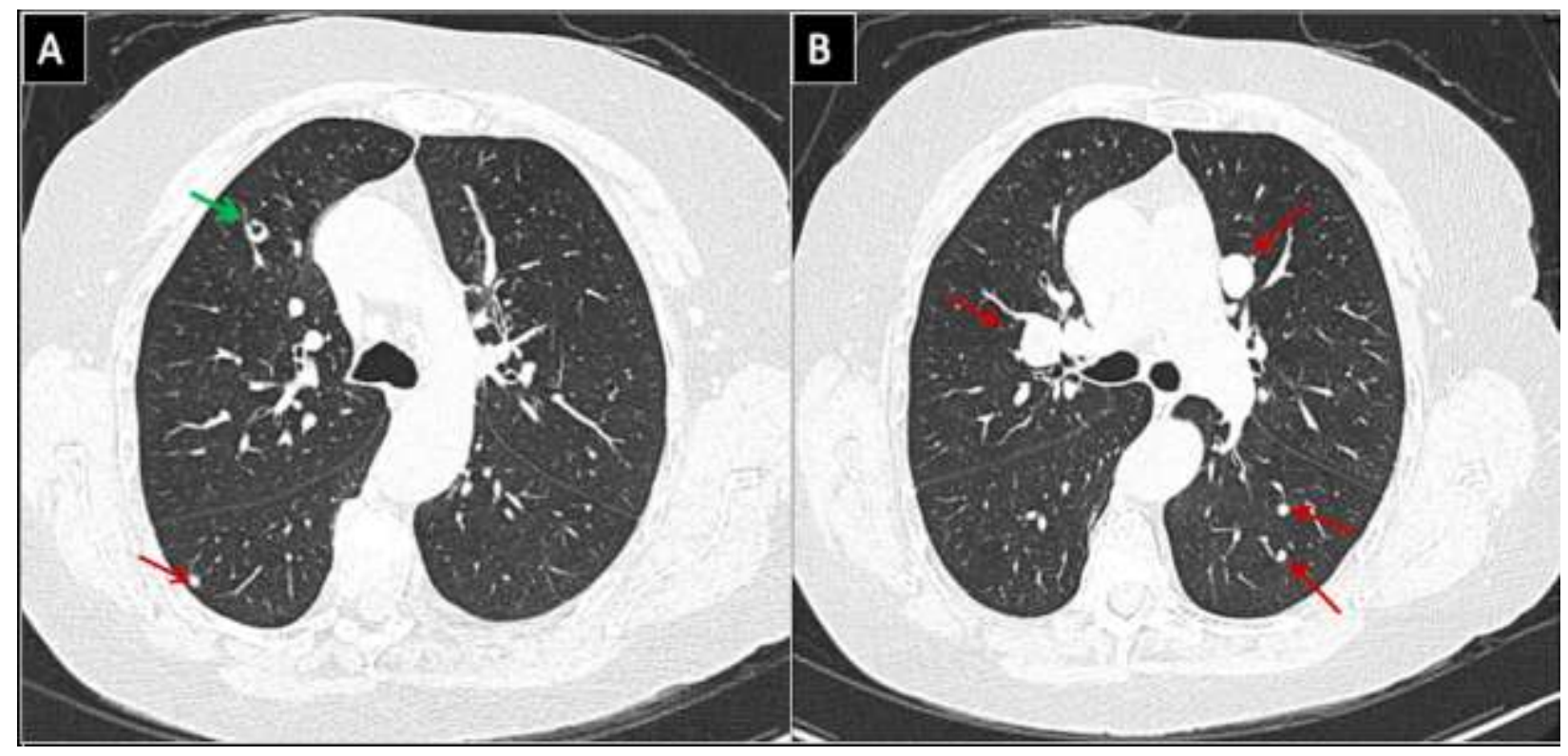

Figure 1:-Computed Tomography (CT) of the lung shows bilateral pulmonary nodules (Red arrows) with one cavitating nodule (Green arrow)

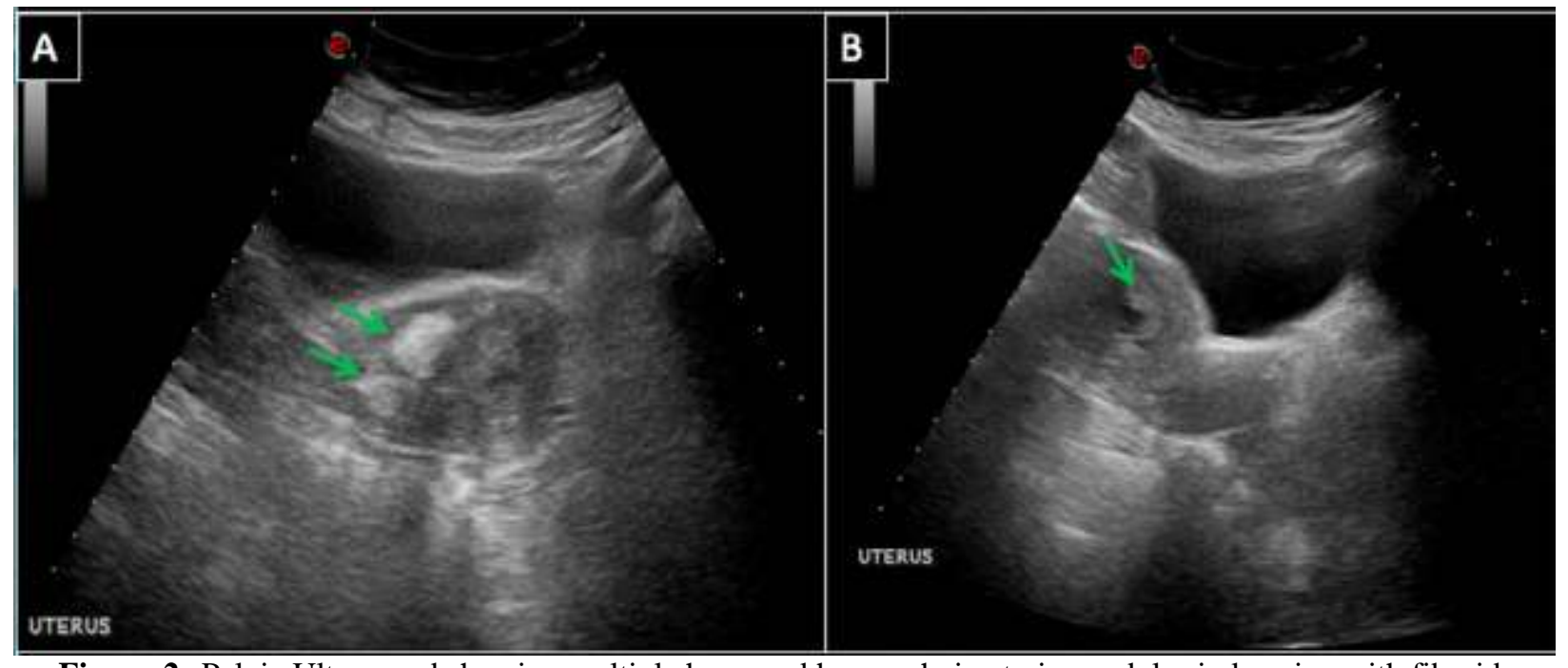

Figure-2:-Pelvic Ultrasound showing multiple hypo and hyperechoic uterine nodules in keeping with fibroids (Green Arrow). 


\section{References:-}

1. Barnaś E, Książek M, Raś R, Skręt A, Skręt- Magierło J, et al. (2017) Benign metastasizing leiomyoma: A review of current literature in respect to the time and type of previous gynecological surgery. PLOS ONE 12(4): e0175875.

2. Abramson S, Gilkeson RC, Goldstein JD, Woodard PK, Eisenberg R, Abramson N: Benign metastasizing leiomyoma: clinical, imaging, and pathologic correlation. Am J Roentgenol 2001, 176:1409-1413.

3. Patton KT, Cheng L, Papavero V, et al. Benign metastasizing leiomyoma: clonality, telomere length, and clinicopathologic analysis. Mod Pathol. Jan 2006;19(1):130-140.

4. Chen S, Zhang Y, Zhang J, et al. Pulmonary benign metastasizing leiomyoma from uterine leiomyoma. World J Surg Oncol. 2013;11:163.

5. Alselwi, W., Alfotih, S., Azam, F. and Alwabari, A. (2016). Benign Pulmonary Metastasizing Leiomyoma: A Case Report. American Journal of Cancer Case Reports, 4(2), pp.1-5.

6. Novel hormone treatment of benign metastasizing leiomyoma: an analysis of five cases and literature review. Lewis EI ${ }^{1}$, Chason RJ, DeCherney AH, Armstrong A, Elkas J, Venkatesan AM.

7. Rivera JA ${ }^{1}$, Christopoulos S, Small D, Trifiro M. Hormonal manipulation of benign metastasizing leiomyomas: report of two cases and review of the literature. J Clin Endocrinol Metab. 2004 Jul;89(7):3183-8.

8. Ponea AM, Marak CP, Goraya H, Guddati AK. Benign metastatic leiomyoma presenting as a hemothorax. Case Rep Oncol Med. 2013;2013:504589. 